Syracuse University

SURFACE

SU Faculty publications on Wellness

$3-2013$

\title{
A Comparison of Noncontingent Plus Contingent Reinforcement to Contingent Reinforcement Alone on Students' Academic Performance
}

Carlos J. Panahon

Minnesota State University

Brian K. Martens

Syracuse University, bkmarten@syr.edu

Follow this and additional works at: https://surface.syr.edu/wellness

Part of the Psychology Commons

\section{Recommended Citation}

Panahon, Carlos J. and Martens, Brian K., "A Comparison of Noncontingent Plus Contingent

Reinforcement to Contingent Reinforcement Alone on Students' Academic Performance" (2013). SU

Faculty publications on Wellness. 1.

https://surface.syr.edu/wellness/1

This Article is brought to you for free and open access by SURFACE. It has been accepted for inclusion in SU Faculty publications on Wellness by an authorized administrator of SURFACE. For more information, please contact surface@syr.edu. 
This is an author-produced, peer-reviewed version of this article. The published version of

this document can be found online in the Journal of Behavioral Education ( doi:

10.1007/s10864-012-9157-x) published by Springer. The official page numbers are noted in

brackets throughout the article.

\title{
A Comparison of Noncontingent Plus Contingent Reinforcement to Contingent Reinforcement Alone on Students’ Academic Performance
}

\author{
Carlos J. Panahon, Minnesota State University \\ Brian K. Martens, Syracuse University
}

Keywords: Noncontingent reinforcement, Contingent reinforcement, Reinforcement-based programs Math problems

\begin{abstract}
Noncontingent reinforcement (NCR) can be described as time-based or response-independent delivery of stimuli with known reinforcing properties. Previous research has shown NCR to reduce problem behavior in individuals with developmental disabilities and to interfere with the acquisition of more desired alternative behavior. To date, however, little research has examined the effects of NCR on children's academic performance. The present study examined the effects of NCR on the completion of math computation worksheets by 3 students in an elementary school setting. An ABCB reversal design was used to compare an NCR schedule in combination with contingent reinforcement to contingent reinforcement alone. Results showed that digits correct per session decreased to baseline levels for all 3 students during implementation of the NCR plus contingent reinforcement condition. Implications of these results for the reductive effects of NCR are discussed.
\end{abstract}

\section{Introduction}

Noncontingent reinforcement (NCR) can be described as time-based or response-independent delivery of stimuli with known reinforcing properties (Vollmer et al. 1993). With an NCR schedule, reinforcers are delivered based on a predetermined time schedule (e.g., every 20 s) rather than being influenced by the person's [end of page 37] behavior. For example, a reinforcer may be delivered every $30 \mathrm{~s}$ regardless of whether the individual exhibited the targeted behavior or completed the required amount of work.

NCR has been shown to reduce severe problem behavior in individuals with developmental disabilities (Marcus and Vollmer 1996; Vollmer et al. 1993; Vollmer et al. 1995) and to interfere with the acquisition of an alternative behavior (Goh et al. 2000). Marcus and Vollmer (1996) investigated the efficacy of combining NCR with an intervention that replaced challenging behaviors by teaching functionally equivalent communication responses (i.e., mands). Three children with developmental disabilities participated in the study. All participants engaged in high rates of challenging behavior maintained by access to preferred tangible items. Results showed that the initial NCR schedule suppressed the children's inappropriate behavior. When a differential reinforcement of alternative (DRA) behavior condition was added to an already thinned NCR schedule, NCR did not preclude the establishment and maintenance of mands. It should be noted, however, that manding increased only after the NCR schedules were thinned. It is possible that the initial dense NCR schedules for both children may have interfered with the acquisition of alternative behavior.

Goh et al. (2000) attempted to extend the work of Marcus and Vollmer (1996) by examining the extent to which both rich and thinned NCR schedules might interfere with differential reinforcement contingencies designed to strengthen alternative behavior. Two women with developmental disabilities exhibiting self-injurious behaviors (SIB) participated in the study. Participants were exposed to baseline, NCR (dense) plus DRA, and NCR (thinning) plus DRA conditions using a multiple baseline design across participants. Results indicated that NCR (dense) plus DRA decreased SIB, but there was little or no increase in mands. After the NCR schedule was thinned, SIB remained low but mands increased. Neither of the participants showed evidence of mand acquisition while the rich NCR schedule was in effect.

The results of these studies suggest that NCR may reduce the effectiveness of reinforcement-based programs. Marcus and Vollmer (1996) argued that response suppression may result from satiation to the reinforcer being delivered. Goh et al. (2000) suggested that response suppression during rich NCR schedules is likely to result from satiation, whereas lean schedules may suppress responses as a result of extinction. That is, NCR may be conceptualized as an extinction procedure because the functional relation between behavior and reinforcement is weakened or eliminated. Hagopian et al. (1994) believed that NCR might alter the reinforcing properties of stimuli 
by eliminating a deprivation state that leads to increased responding. The authors also viewed NCR as an extinction procedure because the contingency between the destructive behavior and the reinforcing consequence was eliminated.

Although considerable attention has focused on the use of NCR to reduce challenging behavior, little research has been conducted on how NCR might affect academic performance in a classroom setting. Martens et al. (2003) conducted the first study to examine the carryover effects of a FT reinforcement history on children's completion of math problems. Two female students diagnosed with [end of page 38] Learning Disabilities participated in an ABABA design. During Choice conditions (A), students were given a choice of completing easy problems reinforced on a lean schedule or completing difficult problems reinforced on a rich schedule. During FT reinforcement conditions (B), reinforcers were delivered on an FT 20-s schedule for eight sessions. The authors were interested in whether exposure to an FT schedule differentially influenced students' choices of difficult or easy problems in subsequent contingent reinforcement conditions. During the FT conditions, students completed both types of problems with higher percentages of errors. Carryover effects from FT sessions were observed for both students in terms of committing more errors and differentially influencing responding to the high effort alternative. That is, the children chose to complete more easy problems on a lean schedule (i.e., the lower effort alternative) for several sessions immediately following the FT condition. In addition, both students showed evidence of response suppression during the FT conditions. This was the first applied study to demonstrate that the suppressive effects of FT reinforcement can carry over to subsequent conditions. However, these effects were very brief for the two participants. Also, individual differences were observed in the participants' sensitivity to the changes in reinforcement schedules.

The purpose of this study was to replicate and extend the findings of previous research (e.g., Goh et al. 2000; Martens et al. 2003) in two ways. First, the present study examined the effects of a fixed-time schedule of reinforcement on students' academic performance. Second, this study explored the effects of NCR with children without cognitive impairment. It was hypothesized that when reinforcement was provided contingent on work completion, increases in the amount of work completed would be observed compared to baseline. Further, it was hypothesized that students' academic performance would decrease when an NCR schedule was presented in combination with contingent reinforcement.

\section{Method}

\section{Participants and Setting}

Three male students enrolled in the same fourth-grade regular education classroom in an urban school district in the Northeast participated in the study. Only male students were recruited to eliminate any possible gender differences. Both Mark and David were African American, and Jason was Hispanic. Mark was 11 years old and had repeated the first and fourth grades. David was 10 years old and had repeated the first grade. Jason was 9 years old. None of the students had been identified as having a disability.

Curriculum-Based Assessment (CBA) results indicated that Mark was at mastery level in sums to 9 and instructional level in sums to 18 as well as two-digit by two-digit addition without regrouping. Jason and David were at instructional levels for sums to 9, sums to 18, and two-digit by two-digit addition without regrouping. All three of the students were at frustrational level for two-digit by two-digit addition [end of page 39] with regrouping. Therefore, the highest instructional level was two-digit by two-digit addition without regrouping for all three participants. All sessions lasted 5 min and were conducted individually 3-4 times a week. Sessions occurred in a separate small room in the back of the participants' classroom. The room contained a desk and chairs for the student and the experimenter(s).

\section{Materials}

\section{Curriculum-Based Measurement (CBM) Probes}

CBM probes were used to identify each student's instructional level in mathematics (Shinn 1989). Each probe consisted of 30 single-skill computation problems (i.e., 6 rows with 5 problems per row). Worksheets varied in difficulty based on the number of digits in each problem. Math computation skills assessed were determined by the teacher reporting the level at which the students were working. Each student was administered two worksheets for each skill assessed (8 total worksheets). Four different addition skills (sums to 9 , sums to 18, two-digit by two-digit addition without regrouping, and two-digit by two-digit addition with regrouping) were assessed.

\section{Reinforcer Preference Survey}


Before the start of the study, the Reinforcer Preference Survey (Northup et al. 1996) was given to the students' teacher. This scale consists of 42 items that are commonly used as reinforcers in a classroom setting (e.g., reward certificate and erasers). The teacher was asked to specify which items were acceptable reinforcers in her classroom. Only items approved by the teacher were presented as reinforce options to the participants. The pool of approved items included reward certificates, stickers, pencils, pretzels, markers, cookies, popcorn, candy, erasers, and puzzles.

\section{Reinforcer Preference Assessment Picture Cards}

Picture cards were created to represent each of the reinforcers approved by the participants' teacher. Each card had a picture of the approved item on one side. Pictures were either computer generated or hand drawn. Each card was 300 9500 in size and laminated.

\section{Experimental Worksheets}

Math worksheets were designed for each student using www.interventioncentral.org, an online database that generated random two-digit by two-digit addition problems. Worksheets consisted of 30 single-skill problems created at the child's highest instructional level as determined by CBM screening. Different color worksheets were used to correspond to each of the different conditions of the study (i.e., white $=$ Baseline, yellow $=$ Rewards, blue $=$ Rewards + Bonuses). [end of page 40]

\section{Preliminary Assessments.}

\section{Reinforcer Preference Assessment}

A reinforcer preference assessment was conducted for each child using a paired-stimulus pictorial choice assessment procedure (Fisher et al. 1992; Northup et al. 1996). Prior to the assessment, the Reinforcer Assessment Survey (Northup et al. 1996) was given to the participants' teacher to identify acceptable items for use in the classroom. Once a list of possible reinforcers was created, picture cards were designed to represent each one. At the beginning of the assessment, each picture card was shown to the child to ensure that the student was capable of identifying every item. If the child was unable to identify any item from the picture, the real item was presented to the child. The picture card of this item was then presented to the child again. This procedure was repeated until the student identified each item accurately from the corresponding picture.

Picture cards were presented to each participant in a pairwise manner such that each item was paired with every other item. Each picture was presented nine times to the participant for a total of 45 trials. Highly preferred items were identified for each student based on the percentage of trials each item was chosen. The three items with the highest percentages for each child were used as the highly preferred reinforcers for the duration of the study. Jason's highly preferred reinforcers were puzzles, pretzels, and reward certificates. He selected these items during 100,78 , and $67 \%$ of the trials, respectively. Mark's highly preferred items consisted of candy (100\%), popcorn (89 $\%)$, and cookies (78 \%). Lastly, candy (100\%), reward certificates (89\%), and cookies (67\%) were identified as highly preferred reinforcers for David.

\section{CBM Screening}

A CBM screening was conducted with all participants to determine their highest instructional level in math. Instructional level was defined as the computation skill at which students answered 20-39 digits correct per min (DCPM) with 3-7 errors (Shapiro, 2011). The experimenter told the student, "The sheets on your desk are math facts. All of the problems are _ _ (specify math skill). When I say begin, start with the first problem and work across the page (demonstrate by pointing). Then go down to the next row. If you cannot answer a problem mark an $\mathrm{X}$ on it and go to the next one. Are there any questions? Begin." At the end of $2 \mathrm{~min}$, the student was told, "Stop, please put your pencil down." This procedure was repeated until the highest instructional level was determined for each participant.

\section{Response Definitions and Measurement}

The dependent measure was the total number of digits correct completed by the student during each 5-min session. To be counted as correct, a digit had to be the correct answer to a problem placed in the correct column on the worksheet. [end of page 41]

\section{Experimental Design and Procedures}

An ABCB reversal design was used to examine the effects of a fixed-time reinforcement schedule with and without contingent reinforcement on students' digits correct per session. In the baseline condition (A), no reinforcement was 
provided to the students for correct problem completion. During the Rewards condition (B), tokens were delivered for correct math problems according to a fixed-ratio (FR) schedule. A different FR schedule was determined for each participant based on their baseline rates of responding, so that the maximum number of tokens earned during a 5-min session was 20. This number was chosen because it was equal to the number of possible tokens students could receive under the NCR schedule. The FR schedules in effect during the Rewards condition were David FR-6 schedule, Jason FR-7, and Mark FR-9. In the Rewards + Bonuses phase (C), participants were exposed concurrently to an FR schedule of reinforcement and an NCR schedule, in which tokens were delivered every $15 \mathrm{~s}$. At the end of all Rewards and Rewards + Bonuses sessions, participants were able to exchange the tokens earned for preferred backup rewards. For Mark and David, an extra set of Rewards + Bonuses and Rewards conditions was conducted to evaluate whether additional experience with the schedules of reinforcement was needed to influence responding.

\section{Baseline}

At the beginning of each baseline session, a set of white math worksheets and a pencil were placed on the desk in front of the student. The experimenter told the student, "The sheets on your desk are all two-digit by two-digit addition problems without regrouping. When I say begin, you may do as many or as few problems as you like or none at all. You will have $5 \mathrm{~min}$ to work. Are there any questions? Ready, set, begin." No reinforcement or additional prompting was provided during this phase. Baseline sessions were conducted until a stable or decreasing rate of academic performance had been established.

\section{Contingent Reinforcement (Rewards)}

In the Rewards condition, a set of yellow math worksheets and a pencil were placed on the desk in front of the student at the start of each session. The student was told by the experimenter, "The sheets on your desk are all twodigit by two-digit addition problems without regrouping. When I say begin, you may do as many or as few problems as you like or none at all. You will have 5 min to work. Today you will be able to earn a point for every _ math problems you answer correctly. At the end of the session, you can trade in every 4 points for your choice of rewards. (The picture cards representing the highly preferred rewards were placed in front of the student on the desk.) Are there any questions? Ready, set, begin." At the end of the session, the participant was told, "Today you completed 42] problems correctly. You have earned rewards. Therefore, you may now choose rewards.” [end of page

\section{Contingent Reinforcement Plus NCR Schedule (Rewards + Bonuses)}

In the Rewards + Bonuses condition, a set of blue math worksheets and a pencil were placed on the desk in front of the student. The participant was told by the experimenter, "The sheets on your desk are all two-digit by two-digit addition problems without regrouping. When I say begin, you may do as many or as few problems as you like or none at all. You will have 5 min to work. Today you will be able to earn a point for every _ math problems you answer correctly. In addition, you will receive a point every $15 \mathrm{~s}$ for free. You will receive a point whether you decide to work on the math problems or not. Therefore, you will earn 4 points every min just for sitting at the desk. At the end of the session, you can trade in the points you received and earned for your choice of rewards. You can exchange 4 points for a reward. (The picture cards representing the highly preferred rewards were placed in front of the student on the desk.) Are there any questions? Ready, set, begin." At the end of the session, the participant was told, "Today you completed __ problems correctly. You have earned _ _ points. In addition you received 4 points for every minute you sat here. You were seated for $5 \mathrm{~min}$, so you received 20 points for free. Therefore, you have a total of __ points (add earned and received points). You may now choose rewards."

\section{Treatment Integrity and Interscorer Agreement}

Experimenters and observers were four graduate students and one undergraduate student who all had prior experience implementing reinforcement schedules with children in a school setting. Training was conducted with all experimenters before the start of the study. Each experimenter demonstrated proficiency in conducting the sessions prior to working with the participants. Experimenters and observers completed session protocols with $100 \%$ accuracy prior to working with students. Treatment integrity and interscorer agreement (ISA) were calculated for 39 $\%$ of sessions across all participants and conditions.

Protocols were developed for sessions in each condition in order to assess treatment integrity. An independent observer recorded each step completed by the experimenter. Treatment integrity was calculated by dividing the number of steps completed correctly by the number of total possible steps and multiplying by $100 \%$. Treatment integrity was $100 \%$ across all participants and conditions. 
To assess ISA, observers independently counted the number of digits correct on completed worksheets. ISA for digits correct was calculated by dividing the number of agreements by the number of agreements plus disagreements and multiplying by $100 \%$. ISA for Jason, Mark, and David was $99.9 \%$ across all sessions, with respective ranges of $99.3-100 \%, 99.4-100 \%$, and $99.3-100 \%$.

\section{Results}

The mean number of digits correct per session and standard deviations across sessions for each participant and condition are summarized in Table 1. Percentages [end of page 43]

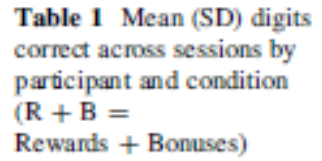

\begin{tabular}{llll}
\hline Conditions & Jason & Mark & David \\
\hline Baseline & $138.7(13.9)$ & $173.0(15.3)$ & $119.7(11.7)$ \\
Rewards & $156.7(8.8)$ & $183.7(9.0)$ & $121.2(7.0)$ \\
R + B & $139.5(18.9)$ & $190.5(23.3)$ & $121.0(7.9)$ \\
Rewards & $148.0(11.3)$ & $229.4(9.8)$ & $137.4(16.5)$ \\
R + B & - & $165.6(24.8)$ & $118.8(3.0)$ \\
Rewards & - & $199.7(27.3)$ & $139.3(9.7)$ \\
\hline
\end{tabular}

of nonoverlapping data (PND) for each participant between conditions are summarized in Table 2. PND is a method of evaluation that is often used to supplement visual inspection of single-subject graphs (Parker and Vannest 2009). PND was calculated by dividing the number of data points in the second condition that did not fall within the value range of the first condition by the total number of data points in the second condition and multiplying by $100 \%$.

The upper panel of Fig. 1 shows the results for Jason. There was a slight decreasing trend in baseline, with a mean digits correct of 138.7. Sessions from the first Rewards condition showed an increasing trend, a slightly higher mean $(\mathrm{M}=156.7)$, and moderate overlap ( $\mathrm{PND}=43 \%)$ with baseline performance. When reinforcement was delivered on both FR and NCR schedules in the Rewards + Bonuses condition, Jason's responding decreased to baseline levels with a decreasing trend throughout the phase $(M=139.5$; PND $=44 \%)$. When the second Rewards phase was implemented, Jason's performance increased to an average of 148.0 digits correct per session but dropped during the last session.

The middle panel of Fig. 1 depicts the results for Mark. Baseline sessions showed a steady state of responding followed by a decreasing trend toward the end of the condition $(M=173.0)$. The first Rewards condition showed a slight increase in mean digits correct $(\mathrm{M}=183.7$; PND $=17 \%)$ over baseline with a stable pattern of responding. An increasing trend in digits correct per session was observed during the first Rewards + Bonuses condition and the second Rewards condition. Results from the second Rewards + Bonuses condition showed a sharp decrease in Mark's responding to an average of 165.6 digits correct per session and a PND of $100 \%$. Results from the third Rewards condition showed that Mark's pattern of responding increased again in response to contingent reinforcement $(\mathrm{M}=199.7)$.

The bottom panel of Fig. 1 shows the data for David. Responding at the beginning of the baseline condition was variable with a decreasing trend $(\mathrm{M}=119.7)$. When the first set of Rewards and Rewards + Bonuses conditions

Table 2 Percentages of nonoverlapping data across participants between conditions

\begin{tabular}{llll}
\hline Conditions & Jason (\%) & Mark (\%) & David (\%) \\
\hline Baseline to rewards & 43 & 17 & 0 \\
Rewards to rewards + bonuses & 44 & 17 & 14 \\
Rewards to rewards + bonuses & - & 100 & 0 \\
\hline
\end{tabular}

\section{[end of page 44]}



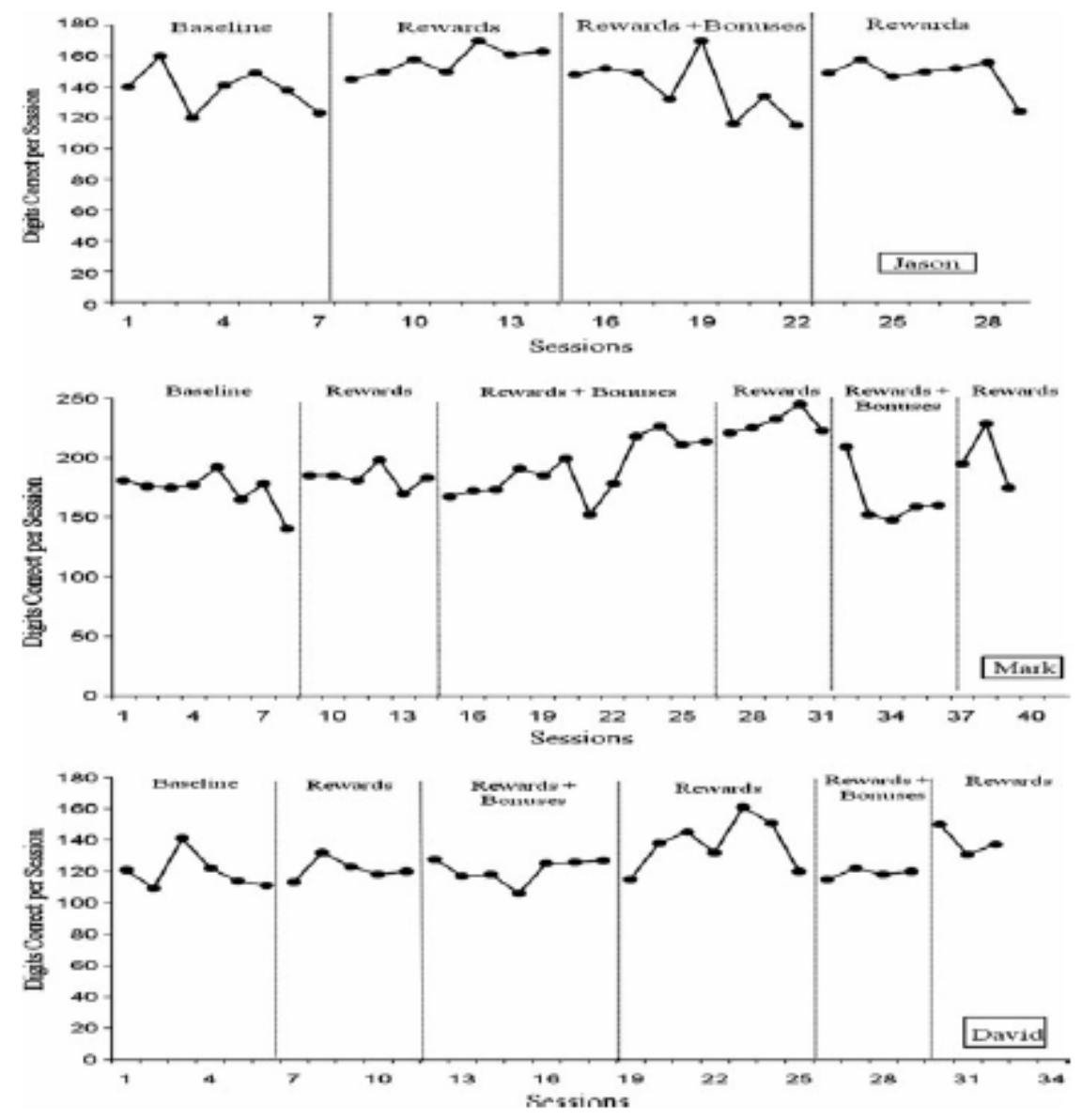

Fig. 1 Digits correct per session across all conditions for Jason, Mark, and David

were implemented, David's pattern of responding was generally undifferentiated $(\mathrm{M}=121.2$ and 121.0; $\mathrm{PND}=0$ and $14 \%$, respectively). Results of the second Rewards condition showed an increase in David's performance $(\mathrm{M}=$ 137.4). Implementation of the second Rewards ? Bonuses condition decreased performance to a mean of 118.8 digits correct but still fell within the lower limit of the adjacent condition (PND $=0 \%$ ). When the final Rewards condition was conducted, David's level of responding increased to a mean of 139.3 digits correct.

\section{Discussion}

The purpose of the present study was to evaluate the effects of NCR on students' academic performance. The findings of this study were mixed and therefore should be viewed as preliminary. It was hypothesized that increases in the number of digits [end of page 45] correct per session would be observed during the Rewards condition in comparison with baseline. Providing reinforcement contingent on correct problems increased performance over baseline during either the initial or subsequent Rewards conditions. Jason's PND of $43 \%$ indicated a stronger effect in comparison with Mark's (PND $=17 \%$ ) and David's (PND $=0 \%$ ) performances during the initial Rewards condition. A second set of Rewards conditions was needed to see an increase in digits correct per session in comparison with baseline for Mark and David.

Further, it was hypothesized that students' academic performance would decrease during the Rewards + Bonuses condition. Jason showed a decreasing trend in the number of digits correct during this condition. However, the reductive effect of NCR was moderate as indicated by the PND of $44 \%$. For Mark and David, drops in their rates of responding were observed but only during subsequent exposures to the Rewards and Rewards + Bonuses conditions. Mark's digits correct decreased sharply during his second Rewards + Bonuses condition (PND = $100 \%$ ). David's level of responding decreased but was still within the lower range of his performance during the previous Rewards condition (PND $=0 \%$ ).

The slight decrease in responding during the Rewards + Bonuses condition for the three children was similar to the reductive effects of NCR on problem behavior found in previous research. Extinction and satiation 
have been provided as two explanations for the suppressive effects of NCR. Vollmer, Marcus, and Ringdahl (1995) reported that NCR might represent an extinction procedure by weakening or eliminating the relationship between the target behavior and reinforcement. Goh et al. (2000) and Marcus and Vollmer (1996) stated that satiation to the reinforce being delivered on rich NCR schedules may account for response suppression of problem behavior. Results from this study support the former extinction explanation. That is, the reinforcing consequences of the FR schedules may have been reduced by delivering the same reinforcers noncontingently to the students during the Rewards + Bonuses condition. Satiation probably did not occur because delivery of rewards was withheld until the token exchange at the end of each session. In addition, satiation probably did not occur because the students were able to choose from their three most preferred items at the end of each session.

It is interesting to note that responding decreased during the Rewards + Bonuses phase for the three students. This change suggests that Jason required five sessions to discriminate between the two conditions. After these initial Rewards + Bonuses sessions, Jason may have determined that he received more reinforcers during this condition even if he did less work. Apparently, Mark and David needed an additional set of manipulations for them to discriminate between the two conditions. During the second Rewards ? Bonuses condition, Mark's responding decreased rapidly and David's responding remained at baseline levels. The difficulty to quickly discriminate between schedules of reinforcement existed even though contrived directions were delivered to the participants at the beginning of each condition.

The decrease in responding during the Rewards + Bonuses conditions in the present study was not as consistent nor as robust across participants as originally [end of page 46] hypothesized. These patterns of responding may be explained both by the sequence of conditions to which the children were exposed and by the design of the Rewards + Bonuses condition. First, the Rewards + Bonuses condition always followed the Rewards only condition. Had the Rewards + Bonus condition followed baseline, participants may have been satisfied with receiving the bonus points and therefore may have responded at lower levels. Second, the students may have interpreted the Rewards + Bonuses condition as a chance to receive more reinforcers than the Rewards only condition because there were two concurrent schedules of reinforcement. This opportunity to receive more tokens may explain the increasing trend observed in Mark's and David's patterns of responding during the first Rewards + Bonuses condition. These students may have been following the rule that if their performance during the Rewards + Bonuses condition was at least equal to their performance during the Rewards condition, they would receive more reinforcers than they had in the previous condition. Also, each student received points during each session and traded in these points for rewards after the session was complete. Therefore, the similarity in the delivery of points during the two conditions may have reduced the discriminability between them.

Another explanation for the inconsistency in results may have been that completing instructional-level math problems alone was reinforcing to the students. Reinforcement received simply for completing math problems may have then competed with the FR and NCR schedules of reinforcement. In addition, high rates of responding during baseline would make it more difficult to see an increase in performance during the subsequent Rewards condition. A final explanation for the relatively small, reductive effects of NCR on students' academic performance may involve the manner in which the NCR contingency was arranged. Specifically, in the Rewards + Bonuses condition, students were told that they would "earn 4 points every min just for sitting at the desk." Although points were delivered independent of work completion, the NCR schedule could be conceptualized as a competing schedule of fixedinterval reinforcement for in-seat behavior. Myerson and Hale (1984) argued that interval schedules compete incrementally with behavior maintained on ratio schedules, and this may have been the case in the present study.

Results of this study may have implications for the importance of implementing reinforcement-based programs with high levels of integrity. In order for these programs to be effective in the classroom, the contingent relationship between behavior and the reinforcing stimuli must be established and clearly explained to students. The contingent relationship is better established when students learn that their behavior will be reinforced consistently. Contingent delivery of reinforcers may be problematic for some teachers-they may omit key steps, fail to consequence target behaviors, or deliver attention for both desired and undesired behavior. When teachers have difficulty delivering reinforcers consistently or contingent on desired student behavior, students may be exposed to NCR schedules in the classroom. This exposure to NCR may reduce the effectiveness of reinforcement-based programs. In addition, students must be able to discriminate the contingent relationship between the target behavior and reinforcement and receiving reinforcement when the target behavior is not exhibited. The decrease in [end of page 47] responding during the Rewards + Bonuses condition demonstrates what can happen when these contingent relations are not clearly established.

Although the current findings extend the NCR literature to children's academic performance, there are several limitations that need to be addressed. First, students were removed from the classroom to conduct the experimental sessions. This may limit generalization of the findings to a classroom situation and a more natural 
school setting. Future research may evaluate the effects of NCR in an actual classroom setting. Second, the amount of variability within phases together with the short length of phases may have limited the findings of the study. Future researchers may want to extend the length of the phases to address this limitation. Third, four of the nine backup reinforcers identified as preferred by the students were chosen less than $80 \%$ of trials during the reinforcer preference assessment. Although students were given a choice of items at the end of each session, the inclusion of some less preferred items may have reduced the effects of the Rewards condition. In addition, the students who participated in the study may have discussed and compared the number of reinforcers received during each session. This may have created competition between the children and contributed to the reinforcing properties of problem completion alone. Future researchers may want to use students from different classrooms to reduce competition. However, it would be difficult to eliminate the possibility of students within the same class or school talking to each other in a natural setting. Also, it is difficult to determine whether the order of conditions had an effect on the target behavior because the Rewards + Bonuses condition always followed the Rewards condition. Future researchers may want to counterbalance the conditions across participants to eliminate any possible sequence effects.

Despite the limitations mentioned above, the results of the present study suggest that providing reinforcement noncontingently may reduce the effectiveness of reinforcement-based programs under some conditions. One interesting question that was not addressed in the present study was the effects of NCR when presented in isolation. In the present study, reinforcement was provided during the Rewards + Bonuses condition through a combination of NCR and contingent schedules. If reinforcement had been provided only on an NCR schedule, perhaps more robust effects on performance may have been observed. Future researchers may want to investigate the effect an NCR alone condition may have on academic performance.

\section{References}

Fisher, W., Piazza, C. C., Bowman, L. G., Hagopian, L. P., Owens, J. C., \& Slevin, I. (1992). A comparison of two approaches for identifying reinforcers for persons with severe and profound disabilities. Journal of Applied Behavior Analysis, 25, 491-498.

Goh, H. L., Iwata, B. A., \& DeLeon, I. G. (2000). Competition between noncontingent and contingent reinforcement schedules during response acquisition. Journal of Applied Behavior Analysis, 33, 195-205.

Hagopian, L. P., Fisher, W. W., \& Legacy, S. M. (1994). Schedule effects of noncontingent reinforcement on attention-maintained destructive behavior. Journal of Applied Behavior Analysis, 27, 317-325. [end of page 48]

Marcus, B. A., \& Vollmer, T. R. (1996). Combining noncontingent reinforcement and differential reinforcement schedules as treatment for problem behavior. Journal of Applied Behavior Analysis, 29, 43-51.

Martens, B. K., Hilt, A., Needham, L. R., Sutterer, J. R., Panahon, C. J., \& Lannie, A. L. (2003). Carryover effects of free reinforcement on children's work completion. Behavior Modification, 27(4), 560-577.

Myerson, J., \& Hale, S. (1984). Practical implications of the matching law. Journal of Applied Behavior Analysis, $17,367-380$.

Northup, J., George, T., Jones, K., Broussard, C., \& Vollmer, T. R. (1996). A comparison of reinforce assessment methods: The utility of verbal and pictorial choice procedures. Journal of Applied Behavior Analysis, 29, 201-212.

Parker, R. I., \& Vannest, K. J. (2009). An improved effect size for single case research: NonOverlap of All Pairs (NAP). Behavior Therapy, 40, 357-367.

Shapiro, E. S. (2011). Academic skills problems: Direct assessment and intervention (4th ed.). New York, NY: The Guilford Press.

Shinn, M. R. (1989). Curriculum-based measurement: Assessing special children. New York, NY: The Guilford Press.

Vollmer, T. R., Iwata, B. A., Zarcone, J. R., Smith, R. G., \& Mazalewski, J. L. (1993). The role of attention in the treatment of attention-maintained self-injurious behavior: Noncontingent reinforcement and differential reinforcement of other behavior. Journal of Applied Behavior Analysis, 26, 9-21.

Vollmer, T. R., Marcus, B. A., \& Ringdahl, J. E. (1995). Noncontingent escape as treatment for selfinjurious behavior maintained by negative reinforcement. Journal of Applied Behavior Analysis, 28, 15-26. [end of page 49] 